\title{
Lightness contrast and failures of constancy: A common explanation
}

\author{
ALAN L. GILCHRIST \\ Rutgers University, Newark, New Jersey
}

\begin{abstract}
Observers were asked to select, from a grid of 16 achromatic Munsell chips presented on a white background in bright illumination, a sample to match a light gray chip simultaneously presented on the same white background but in a shadowed region adjacent to the brightly illuminated region. The border dividing the two fields of illumination was made to appear as either a reflectance edge or an illumination edge by either concealing or revealing the larger context. Each condition thus constituted an experiment on either contrast or constancy, allowing these two phenomena to be compared under comparable conditions. The results indicate that constancy effects are far greater than contrast effects, casting doubt on conventional reductions of the two phenomena to a single explanatory mechanism. Closer analysis of the data indicates that it may be contrast effects and failures of constancy that share a common explanation. Such an explanation, in terms of edge-processing algorithms, is offered and is supported with additional experiments as well as a brief review of previous contrast and constancy findings.
\end{abstract}

Lightness constancy and simultaneous lightness contrast (SLC) have traditionally occupied central roles in the field of lightness perception. Theories have sought not only to relate these two phenomena within the same theoretical structure, but to explain them with the same principle (Freeman, 1967). Constancy and contrast illustrate, in different ways, that a given luminance will vary in lightness (perceived surface reflectance) when it is presented in different contexts. This lack of correlation between luminance and lightness has always seemed significant because of the assumed fundamental role of luminance in the basic response of the visual system (Gilchrist, Delman, \& Jacobsen, 1983; Gilchrist \& Jacobsen, 1984).

Historically, theories of lightness perception have differed, not on the importance of luminance information, but on the role of the context in altering the basic response to luminance. Helmholtz (1868/1962), for example, suggested that the luminance of a surface is meaningless until it is placed (by means of an unconscious inference) within a framework of perceived illumination, and that contrast effects involve the inappropriate use of such frameworks. Helson (1964) operationalized Helmholtz's framework as the weighted average of all luminances in the visual field. The average replaced Helmholtz's cognitive judgment and the weighting handled contrast effects. Wallach (1948) put the luminance of a surface into a ratio with the luminance of the surrounding region as a variable to account for contrast as well as constancy effects.

The direct linkage of contrast and constancy effects reaches its sharpest definition in so-called contrast theories based on neural lateral inhibition. Perceived lightness at any point in the visual field is directly tied to the

Address correspondence to Alan L. Gilchrist, Institute for Cognitive Studies, Rutgers University, Newark, NJ 07102. net amount of neural activity at that point, which is the result of two opposite effects acting on that point: excitation (by light) and inhibition (by neighboring cells). Thus in SLC the two target regions receive equal levels of excitation but different levels of inhibition, producing the contrast effect. In lightness constancy any change in illumination is thought to produce opposite changes in the levels of excitation and inhibition, changes that either exactly (Cornsweet, 1970) or approximately (Jameson \& Hurvich, 1964) cancel each other out, leaving the net level of neural activity constant.

Primarily because of differences in the magnitude of contrast and constancy effects, the reduction of these effects to a common explanation has seemed unlikely to this writer. For example, the lightness illusion in the typical SLC display of two gray squares on black and white backgrounds, although quite intriguing, is really very weak. If the effect were equal in strength to the kind of ratio effects typical of constancy experiments, the gray squares would appear almost white and black (consistent with their target:surround ratios). Nor is the weakness of SLC attributable to the contiguity of the two surrounds, since the same contiguity is present in constancy experiments (Gilchrist et al., 1983; Henneman, 1935; Katz, 1911/1935; Locke, 1935) that yield approximate ratio results. However, this difference in magnitude has been obscured by the fact that contrast and constancy effects have historically been studied in separate experiments under separate conditions. Therefore it seemed important to compare them under comparable conditions. In Experiment 1 these two phenomena were brought together as different conditions of the same experiment, and their juxtaposition under comparable conditions not only highlighted the differences in magnitude of effects (when measured as departures from luminance matches) but also produced a pattern of data that suggests a link between 
contrast effects and failures of lightness constancy. This linkage may provide an edge-processing account not only of lightness contrast effects but of lightness errors in general.

Contrast and constancy experiments typically involve the comparison of two spatially separate target surfaces. In constancy experiments the two targets stand in separate (often side-by-side) fields of illumination (Helson, 1943; Henneman, 1935; Katz, 1911/1935; Locke, 1935), whereas in contrast experiments (when the distinction between reflectance and illumination is present) the target surfaces are often seen against backgrounds of different reflectances (Benary, 1924; Berman \& Leibowitz, 1965; Burgh \& Grindley, 1962; Mikesell \& Bentley, 1930). In Experiment 1, therefore, whether the immediate backgrounds of the target surfaces appeared to differ in reflectance or level of illumination-that is, whether the edge dividing the backgrounds (the mediating edge) appeared as a reflectance edge or an illumination edge-was varied. Since this design qualifies as an example of what Gilchrist et al. (1983) called an edge-substitution experiment, it also serves as a test of whether the visual system responds differently to illumination and reflectance edges.

\section{EXPERIMENT 1}

\section{Method}

Observers. Eight observers served in the constancy condition; 2 additional observers were excused because they reported that the left side of the background appeared as black paper, not as being in shadow. Eight different observers served in the contrast condition and $\mathbf{4}$ additional observers served in the control condition. All were student volunteers and were naive as to the purpose of the experiment.

Apparatus. A large $(46 \times 61 \mathrm{~cm})$ piece of white paper (reflectance $=90 \%$ ) was attached to one wall of a small (1.2 m square) alcove that had been painted matte black in order to control reflected light (see Figure 1). An intense point source of light was positioned at the front of the alcove and a large piece of black paper was suspended from the ceiling of the alcove so that it cast a dark, sharpedged shadow over approximately half of the white paper. The observer stood behind a wall and viewed the array binocularly through a 3-in.-wide horizontal slit in the wall. A baffle prevented the observer from seeing the light source.

A square $\left(3.8 \mathrm{~cm}\left[0.84^{\circ}\right]\right.$ on a side) piece of light gray paper (reflectance $=59 \%$ ) was attached to the shadowed side of the white paper (see Figure 2). This served as the target. On the illuminated side, 16 small squares $\left(2 \mathrm{~cm}\left[0.44^{\circ}\right]\right.$ on a side) of Munsell paper (ranging from black to white) were arranged in a grid.

Conditions. Three conditions were run. In the first, the constancy condition, the observers viewed the display and were asked to describe the display and then select a square from the grid that matched the apparent shade of gray of the target square.

In the second condition, the contrast condition, a large sheet of black paper containing a rectangular aperture $22 \mathrm{~cm}\left(9.7^{\circ}\right)$ wide $\times 13 \mathrm{~cm}\left(5.7^{\circ}\right)$ high was suspended in front of the alcove (Figure 2B). This sheet obscured the observer's view of a large part of the alcove but permitted the observer to see the target square, the grid, and most of their respective surrounds. However, with the loss of context information the shadow now appeared as black paper. The observers viewed the display through this mask and selected a matching sample, as in the first condition.

A third condition, the control condition, was required to control for changes in luminance created by the presence of the mask. Within the rectangular aperture of the mask, of course, all luminances were held constant across conditions. But the mask itself had a luminance $(0.24 \mathrm{fL})$ that was higher than that of the shadowed part of the alcove wall $(0.14 \mathrm{fL})$ and lower than that of the lighted part of the alcove wall $(2.3 \mathrm{fL})$. Such differences could conceivably produce remote contrast effects in the same direction as the expected effects of the independent variable (the perceived nature of the mediating edge). (The problem is limited by the fact that neither of the two remote background regions [0.14 and $2.3 \mathrm{fL}]$ was closer than $2.5^{\circ}$ of visual angle to either the target or the comparison grid, and lateral inhibition effects are thought to be quite small at those separations (Fry \& Alpern, 1953; Leibowitz, Mote, \& Thurlow, 1953). In addition, luminances in other parts of the remote background were altered substantially by the mask in a direction opposite that described above.)

In the control condition, the illumination on the mask was reduced and the right half of the mask was covered with white paper. These measures reversed the direction of any potential remote contrast effects, as can be noted in Figure $2 \mathrm{C}$, since the mask contained luminances that were lower on the left and higher on the right than the luminances it replaced.

Instructions. In each condition the observers were asked to

match the small square on the left to one of the squares in the grid in terms of shade of gray. In other words, if you were to pick up the square on the left and place it right next to the grid, which chip in the grid would match it exactly in shade of gray?

To avoid brightness (as opposed to lightness) matches, the experimenter pointed out the difference between a white surface in shadow and a gray surface, using materials other than those used in the experiment. In general such explanations were given only when the question was raised by an observer, the task being quite clear to most of the observers.

\section{Results}

The Munsell matches are shown in Figure 3. For the constancy condition the median Munsell match was 7.5 $(S D=1.16)$, whereas in the contrast condition it was 3.0 $(S D=0.88)$, a highly significant difference $[t(8)=7.52$, $p<.001]$. The control condition, which was also significantly different from the constancy condition [t(10) $=6.43, p<.001]$, yielded the same median $(3.0$, $S D=0.25)$ as the contrast condition.

\section{Discussion}

Both constancy and contrast effects are conventionally measured in terms of the degree of departure from a luminance match, which in all three conditions of the present experiment would be represented by a Munsell match of 2.1. Thus the constancy effect (5.4 Munsell units) was six times as large as the contrast effect (0.9 Munsell units). This is a very large difference, particularly in view of the fact that the main part of the retinal image (the $9.7^{\circ} \times 5.7^{\circ}$ region seen within the reduction screen) was identical in the two conditions. And the control condition shows that the larger context that was either concealed or revealed by the reduction screen exerted its effect on the data not by the addition or subtraction of luminances per se (with an attendant increase or decrease in inhibition), but rather by producing a perceptual reorganization of the visual field.

These results constitute a serious challenge to the attempt, implicit in virtually all current theories of light- 

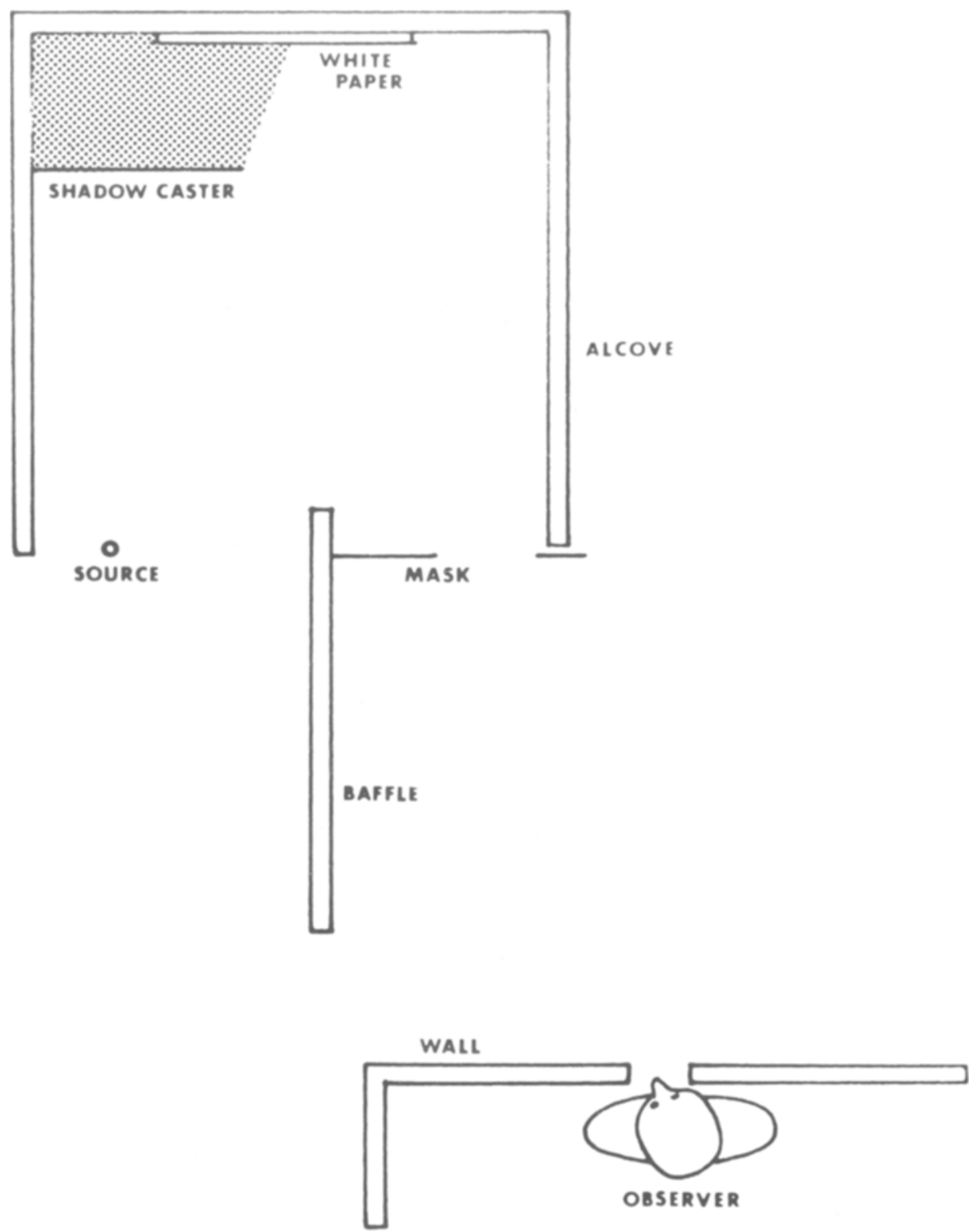

Figure 1. Laboratory arrangements for Experiment 1.

ness perception, to account for contrast and constancy effects directly with the same principle. Given that lightness constancy effects were so much larger than contrast effects under comparable target:surround luminance ratios in the present experiment, it does not seem reasonable to use the same explanation for both. One might say that the existing theories (with the possible exception of that of Helmholtz, 1868/1962) can be applied to constancy or to contrast, but not to both. But the problem runs deeper than this. All of the above theories, except that of Helmholtz, are based on luminances, and since the luminances were held constant across the two conditions, it seems that those theories must make the same prediction in both conditions. Thus it may not be possible to account for the differences obtained in the present experiment with theories based on the processing of absolute amounts of light.

\section{CONSTANCY AND CONTRAST FROM A RELATIONAL PERSPECTIVE}

In recent years, different lines of research have converged on a few basic ideas that form what might be called a relational approach to lightness perception based on edges, or gradients, of light. Two closely related principles seem basic to this approach:

1. Information is extracted from edges in the retinal image. Research on stabilized retinal images (Barlow, 1963; Krauskopf, 1963; Yarbus, 1967) has shown that relative displacement between the retina and the optical image is an absolutely essential condition for visual experience (apart from flickering displays). This fact closely supports the belief that visual cells are stimulated only by a change in light, not by light per se. If this is correct, it means 


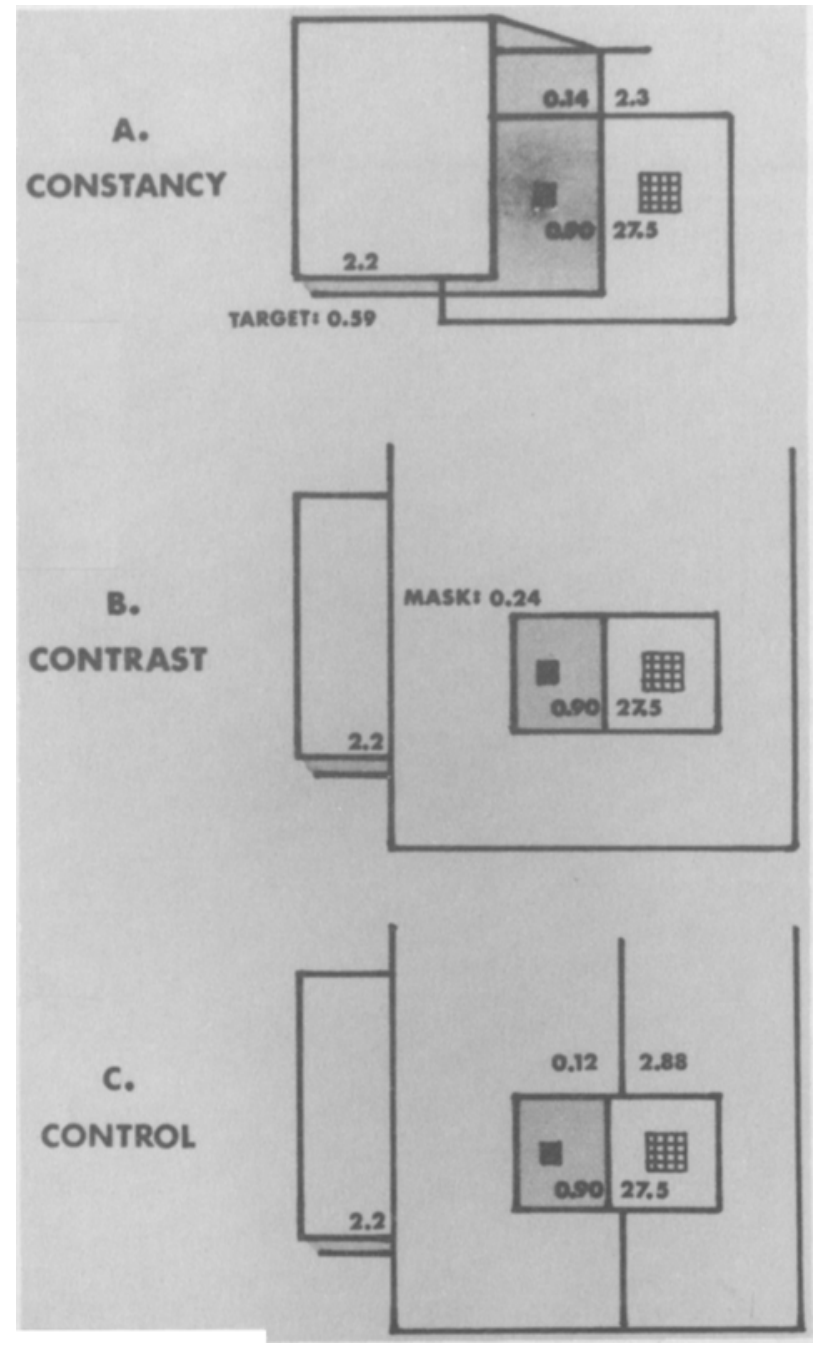

Figure 2. Displays as seen by the observers in Experiment 1, showing luminances in footlamberts. that the basic information is being extracted from edges or gradients in the image and that (barring voluntary eye movements) the homogeneous regions of the image (which already constitute stabilized images, as it were) are "filled in" by default (Krauskopf, 1963).

2. Edge information is strictly relative. It appears that the kind of information produced by retinal stimulation by edges is strictly relative, containing no information as to the absolute luminance (or wavelength) of light on either side of the edge (Whittle \& Challands, 1969). Not only is this entirely consistent with Wallach's (1948) fundamental discovery that equal center:surround ratios produce equal perceived shades of gray, but it suggests a process even simpler than Wallach's (1976) suggestion that luminances are first picked up and only then centrally compared.

The concept of strictly relative information is best illustrated by a simple beam balance. To find the weight ratio between any two objects, one has only to suspend the two objects from opposite ends of the beam and then slide the fulcrum along the beam until a perfect horizontal balance of the beam is achieved. At that point the weight ratio is directly represented in the ratio of the distance of one object from the fulcrum to the distance of the other object. At no point does this process involve any absolute weight information. A pair of objects much heavier (or lighter) than the original pair but standing in the same weight ratio to each other could be substituted without disturbing the balance.

\section{Additional Processes}

Real-world lightness perception requires more than just relative edge information, and Gilchrist (1979; Gilchrist et al., 1983) has proposed two hypothetical processes designed to bridge the gap between edge information and the facts of visual experience:

1. Edge integration. If surface lightness were simply determined by luminance ratios at edges, a white surface

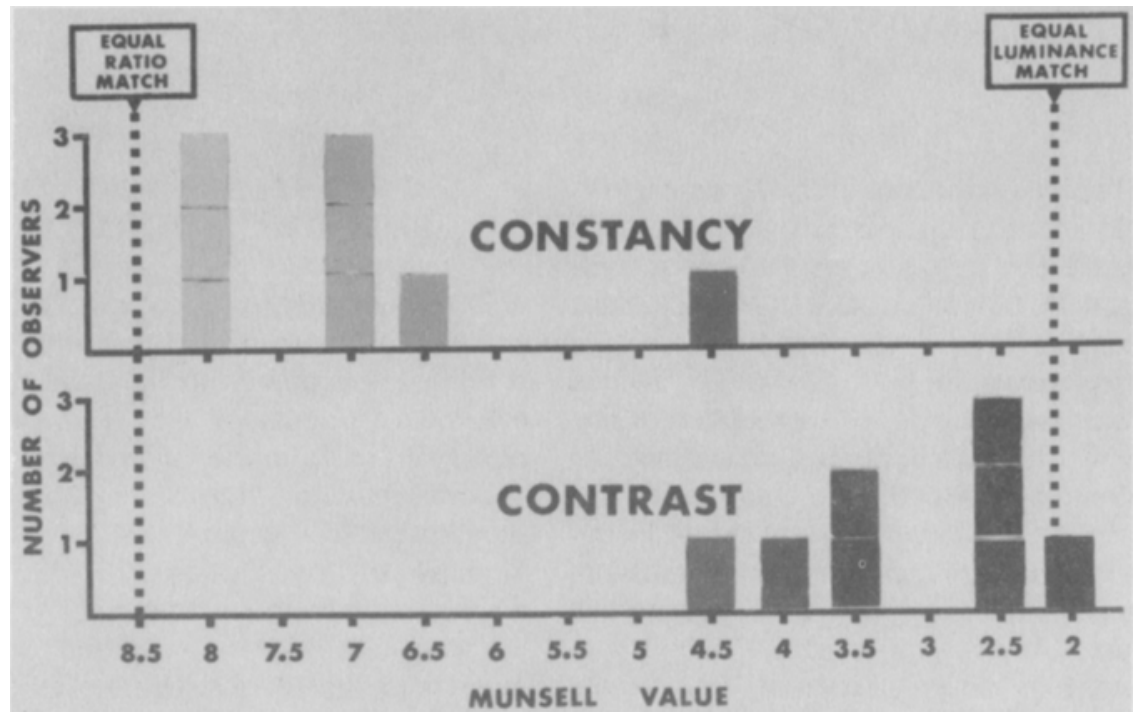

Figure 3. Munsell matches chosen in the constancy and contrast conditions of Experiment 1. 
on a gray background would appear identical to a gray surface on a black background-yet this does not occur. Conversely, objects would appear to change dramatically in surface color as they were viewed against different backgrounds-but in Experiment 1, contrast effects produced by changing backgrounds were far smaller than what one would expect based on the change in edge ratios. A number of researchers (Arend, 1973; Gilchrist et al., 1983; Land \& McCann, 1971; Yarbus, 1967) have dealt with this problem by postulating a kind of edgeintegration process whereby the luminances of spatially remote surfaces can be compared with each other by mathematically integrating all of the changes in luminance (edges) that occur between the two objects (within the image, of course). The results of Experiment 1 support this idea: the lightness of a given target changed dramatically from the constancy condition to the contrast condition, and this change was produced by manipulating the appearance of the mediating edge, not the local edge of each target.

2. Edge classification. Lightness constancy directly implies that changes in illumination are not mistaken for changes in surface reflectance. In the context of an edge approach, this requires some process whereby edges can be classified according to whether they represent changes in illumination or in reflectance, and differentially processed. Presumably, surface lightness is based on an integration of all reflectance edges, whereas our perception of the illumination is based on the integration of illumination edges (it is assumed that this analysis can embrace both spatial and temporal edges). This concept is also supported by Experiment 1, since changes in the larger context were designed to alter the perceptual classification of the mediating edge, which in turn produced a change in the perceived lightness of the target.

What factors determine the classification of an edge? Gilchrist (1979; Gilchrist et al, , 1983; Gilchrist \& Jacobsen, 1984) has echoed Koffka's claim that "a complete answer to this question would probably supply the key to the complete theory of colour perception in the broadest sense" (Koffka, 1935, p. 248). Although this topic lies beyond the scope of the present study, some comments can be offered regarding the classification of the mediating edge in Experiment 1. First, the frequently mentioned factor of edge sharpness, or penumbra, can be ruled out because the mediating edge was sharp in all conditions. One factor that probably played a critical role was the nature of the intersection where the vertical mediating edge crossed the upper horizontal edge of the white background paper. This kind of intersection, in which an illumination edge crosses a reflectance edge, possesses a property that may be called ratio-invariance; that is, the luminance ratio along each edge remains the same even as it crosses the other edge and absolute luminances change. What is important, and what has not been previously recognized, is that this property does not occur when two illumination edges cross one another. In such cases, edge ratios change markedly, but here there is another kind of regularity, difference-invariance. The luminance difference (not ratio) at each edge remains the same across the intersection.

It seems intuitively likely that the mathematical regularities at these intersections, which occur throughout natural scenes, act to constrain the perceptual organization, including classification of edges, of these scenes.

Note that the constraining power of the ratio-invariant intersection is limited, suggesting only that two different types of edges are crossing, but not telling which is which. As far as this constraint alone is concerned, it is possible that, in Experiment 1, the white background paper is actually a field of special illumination (making its boundary an illumination edge), with the shadow appearing as a black surface within a white alcove. However, this organization would encounter various possible contradictions within the larger context, such as any cues indicating that the alcove was black, not white.

Another factor in edge classification may be the systematic relationship between the boundaries of the shadow, which also ran across the ceiling, and the shadow caster. If the mediating edge were seen as a reflectance edge these regularities would be merely coincidental, a strongly nonpreferred outcome, as noted by Rock (1983). These and other factors in edge classification stand in urgent need of further study.

The principles of edge integration and classification go far toward a solution to the classic problem of lightness constancy. The concept that edge ratios are directly picked up through eye movements fits the facts of constancy; as Wallach (1948) showed, crucial edge ratios remain constant even as illumination changes. Edge integration provides for further perceptual stability, especially in the face of changes in the background. And edge classification accounts for lightness constancy where multiple levels of illumination are present within the same visual field (see Gilchrist \& Jacobsen, 1984).

Lightness contrast effects, however, have not yielded readily to this kind of approach. ${ }^{1}$ In fact, it seems that failures of veridicality in general provide the main difficulties. The extraction/classification/integration model presented above essentially predicts veridical perception. According to the theory as developed so far, lightness constancy should be perfect, with neither contrast effects nor failures of lightness constancy. What follows is a systematic attempt to account for SLC within the same relational framework that works so well for constancy. Consider the following potential approaches:

1. Local edge theory. The simplest kind of edge theory would be the view that the lightness (or color) of a surface is determined simply by the change in light at its boundary. Such a theory would nicely explain the direction of the effect in SLC, but it would predict contrast effects far in excess of those actually obtained. The gray squares would appear virtually white and black, which does not happen. Note, for instance, that the main results of Experiment 1 directly contradict a local edge theory. Such a simple account cannot work. 
2. Edge integration. The edge integration concept provides a good explanation for why the two gray squares appear so similar although their edge ratios are so different. In fact, the explanation is too good, since no room is left for any perceived difference; according to edge integration, the squares should look identical. This does represent an advance over the local edge theory, in that the perceived squares are much closer to identical than they are to black and white. One might think about contrast as the result of some small failure of the integration process, but what are the conditions of such a failure?

3. Edge enhancement. Lateral inhibition has often been presented as a mechanism that sharpens the contrast at gradients, making subtle edges more visible, but producing contrast effects as a byproduct (e.g., Cornsweet, 1970). The concept of edge enhancement, however, has so far been presented within a photometer-based framework involving processes of excitation and inhibition. This framework would not qualify as an edge theory, according to the earlier discussion, because, in this framework, the perceived lightness of a given point is defined not by the change in light at an enclosing contour, but by the net amount of excitation and inhibition acting at that point itself. Yet it may be possible to borrow the concept, particularly in view of its emphasis on edges.

4. Edge integration plus edge enhancement. It should be noted at the outset that the combination of edge integration and edge enhancement solves nothing if all edges are enhanced by the same proportion. If all the edge ratios in the SLC pattern were doubled, for instance, the two target squares would still come out as identical, according to the integration process (apart from whatever other effects might be produced). Edge enhancement can be a valid theory only if some edges are enhanced more than others.

What could the rule be? If small changes in light (ratios closer to 1) were enhanced proportionately more than large changes, the predicted results would fit those obtained in the traditional display. This is appealing, especially considering the apparent adaptive value of a system that exaggerates small differences. However, the model runs into trouble when the contrast display is changed. For instance, in the case of two decrements (say, one dark gray target on a white background and one dark gray target on a medium gray background), the model would predict that the target on the lighter (white) background would appear lighter than the other target. This seems counterintuitive.

There is a model that seems to work if, instead of defining the amount of enhancement by the size of the edge, we define it structurally, in terms of the location of that edge in the total gestalt. In the traditional display, this means that the local edges of the target would be enhanced more than the mediating edge would be. This model would also make sense in the doubled-decrement case. This model was tested in Experiment 2 by comparing the results of a variety of contrast patterns.

\section{EXPERIMENT 2}

The five displays used in this experiment are shown in Figure 4. In addition to the traditional display there were a double-decrement pattern and a double-increment pattern, primarily for testing the point discussed immediately above. A display in which equal targets on equal backgrounds were placed on black and white backgrounds was included for its bearing on the edge integration concept. Without edge integration one would expect only the smaller gray backgrounds to show a contrast effect; the targets would be unaffected. With edge integration, however, one would expect these targets to show as much contrast as they would when standing alone on the white and black backgrounds. The fifth pattern was a display used by Wertheimer (see Benary, 1924) to demonstrate the role of perceptual organization in producing the contrast effect.

\section{Method}

The five contrast displays were arranged in a horizontal row against the front wall of a room. They were spaced approximately $7 \mathrm{~cm}$ apart and were viewed under approximately $13.7 \mathrm{fL}$ of illumination. The displays were presented simultaneously to a class of 39 undergraduate students who were naive as to the purpose of the study. A $9 \times 70 \mathrm{~cm}$ Munsell chart containing $163.5-\mathrm{cm}$ square Munsell chips was attached to the wall approximately $0.8 \mathrm{~m}$ above, and centered with respect to, the displays. Viewing distances ranged from 3 to $7.9 \mathrm{~m}$. The observers were asked to select samples from the Munsell chart that matched the apparent shade of gray of each of the targets in each of the displays.

\section{Results}

The results are shown in Figure 4 and Table 1 . The obtained contrast effects (difference between means for target squares were $.64, .92,0, .95$, and .50 Munsell units for Displays A through $E$, respectively.

\section{Discussion}

The results for Display D, which were not significantly different from those for Display A $[t(37)=2.16$, $p>.001$, provide further support for the edge integration concept in that the effect of the white and black backgrounds on the local backgrounds (of equal luminance) of the targets was transferred in turn to the targets themselves. The effect here is quite similar in its logic to one obtained by Arend, Buehler, and Lockhead (1971), who used a Craik-O'Brien contour as a mediating edge.

The results for Display B seem to rule out the theory that small edge differences are enhanced more than large ones, inasmuch as the very direction of the contrast effect is opposite to the predictions of such a model. It is surprising that no contrast effect occurred in the doubleincrement case (Display C). No explanation of this result can be offered here except to note that increments have been shown to produce good luminance matching in other work (Heinemann, 1955; Jacobsen \& Gilchrist, 1988a, 


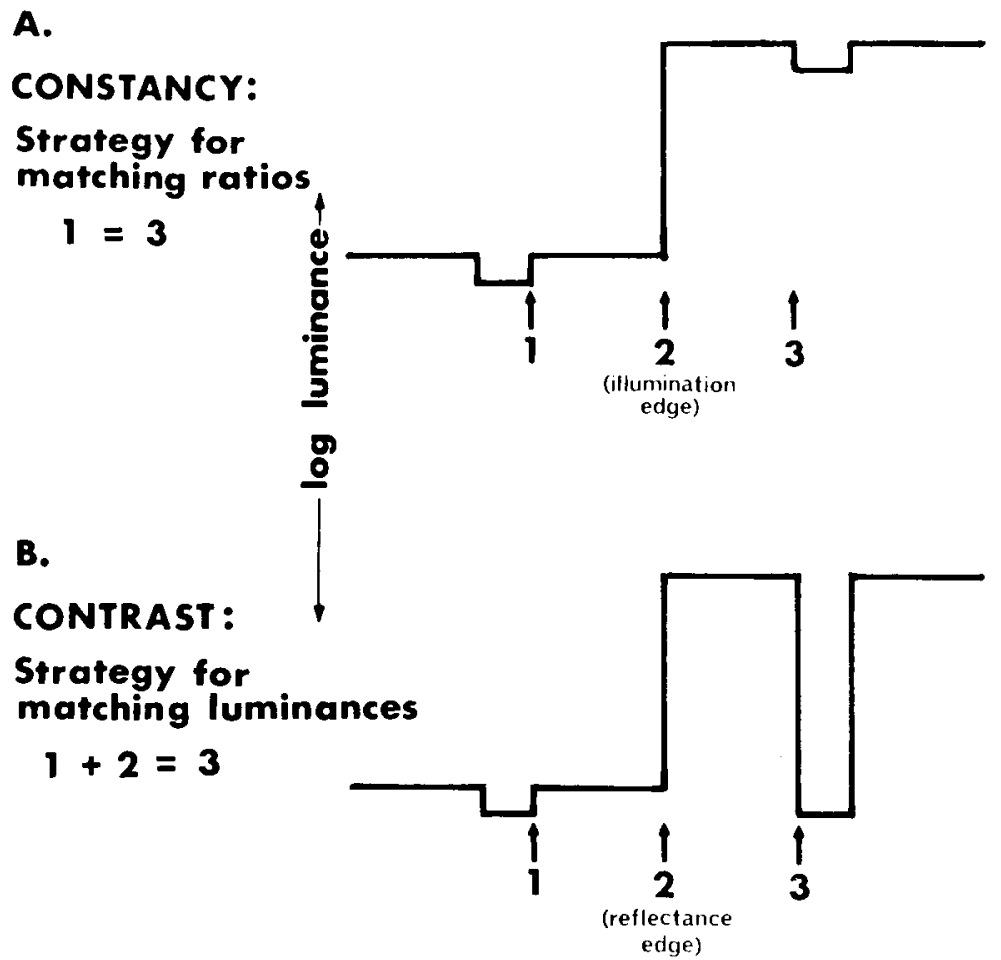

Figure 4. Configurations (showing luminances in footlamberts) and luminance profiles (showing reflectances of mean Munsell matches) for the five displays of Experiment 2.

1988b). The contrast effect was absent only in the group means, however. Approximately a third (14) of the observers saw the left target as lighter, a third (13) saw the right target as lighter, and a third (12) saw them as the same. Perhaps two opposing effects were at work here.

As for an edge-based account of contrast effects, the model most consistent with these displays is the structural model, which posits that local edges get proportionately more weight than mediating edges. As noted before, the term mediating edge cannot be defined apart from the explicit recognition of perceptual structure in the case of displays that have an obvious hierarchical organization. In the side-by-side displays each target belongs to its own background, and the two backgrounds are in turn coequal figures on a common larger background. This implies that the two targets are not compared with each other directly, as they might be in a photometer-based model. Rather, each target is seen in relationship to its background, and only through the additional comparison of the backgrounds can the targets, indirectly, be compared. In fact, contrast effects of these magnitudes ( 0.5 to 1.0 Munsell steps) emerge only in visual fields of sufficient organization.

These points are illustrated in the Benary pattern of Display E. This effect seriously undermines conventional structure-blind accounts of lightness contrast. Consistent with the gestalt account offered here, it shows the importance of perceptual structure even in these contrast effects that have typically been considered to be low level, sensory-type effects. The fact that the results for Display E did not differ significantly from those for Display A [ $t(37)$ $=0.87, p>.001]$ suggests that the Benary effect is the same phenomenon as SLC.

This fits well with Johansson's (1964) observation that the relative component in motion perception is always more visible than the common component, because the mediating edge, by the present definition, is always the boundary of a larger area than the local edge. One of the intriguing features of this model is that it may be equally capable of explaining failures of lightness constancy. Consider how it would account for the data of Experiment 1, in which these two types of perceptual error occurred in the constancy and contrast conditions of the experiment.

\section{PERCEPTUAL ERRORS AND COMPETING OPERATIONS}

Perceptual errors and competing operations. Recall that in Experiment 1 a constancy effect about six times as large

Table 1

Mean Munsell Matches for Experiment 2

\begin{tabular}{ccccccc}
\hline & \multicolumn{2}{c}{$M$} & & \multicolumn{2}{c}{$S D$} & \\
\cline { 2 - 3 } Display & Left & Right & & Left & Right & Significance \\
\hline A & 6.6 & 5.9 & & 0.73 & 0.65 & $t(37)=5.81, p<.001$ \\
B & 3.5 & 2.5 & & 0.50 & 0.56 & $t(37)=12.2, p<.001$ \\
C & 6.6 & 6.6 & & 0.83 & 0.71 & $t(37)=0$, n.s. \\
D & 6.7 & 5.6 & & 0.74 & 0.67 & $t(37)=6.85, p<.001$ \\
E & 5.9 & 6.4 & & 0.50 & 0.64 & $t(37)=4.86, p<.001$ \\
\hline
\end{tabular}


as the contrast effect was obtained, both being measured as departures from luminance matching (appropriate definitions under the photometer metaphor) (see Figure 3). Note, however, that the magnitude by which constancy failed $(8.5-7.5=1.0$ Munsell step) is almost exactly the same as the magnitude of the contrast effect (3.0 $2.1=0.9$ Munsell step), although opposite in sign. Perhaps this is just a coincidence, or perhaps it is a clue.

Assuming that only relative luminance information from edges is available to the system, but that the system is capable of both classifying and integrating these edges, consider what operations would be necessary to arrive at the correct match in the constancy and contrast conditions of Experiment 1. These are illustrated in Figure 5. In the constancy condition, given that the mediating edge (edge 2) is an illumination edge, it should not be included in the edge integration, and a matching square should be selected that stands in the same relationship to the righthand background as the standard target stands to the lefthand background. In other words, the ratio at edge 3 should be equal to the ratio at edge 1 .
In the contrast condition, on the other hand, the intervening edge is perceived as a reflectance edge (although it is physically an illumination edge), and thus should be included in the edge integration. Since illumination is perceived as uniform, reflectance is completely correlated with luminance, and therefore the appropriate matching square will be the one with the same luminance as the target square. This will occur when edge 3 is equal to the combination of edges 1 and 2 .

The actual data obtained in Experiment 1 show that neither the ratio matching operation nor the luminance matching operation is carried out completely; each, however, is approximated. Putting it another way, each condition calls for a different type of equality; ratio matching in the constancy condition requires that edge 2 (as an illumination edge) be fully omitted from the integration process, whereas luminance matching in the contrast condition requires that edge 2 (as a reflectance edge) be fully included in the integration process. Here is where the failure seems to occur. The data in Figure 3 tend strongly toward these poles, but the shortfall (approximately

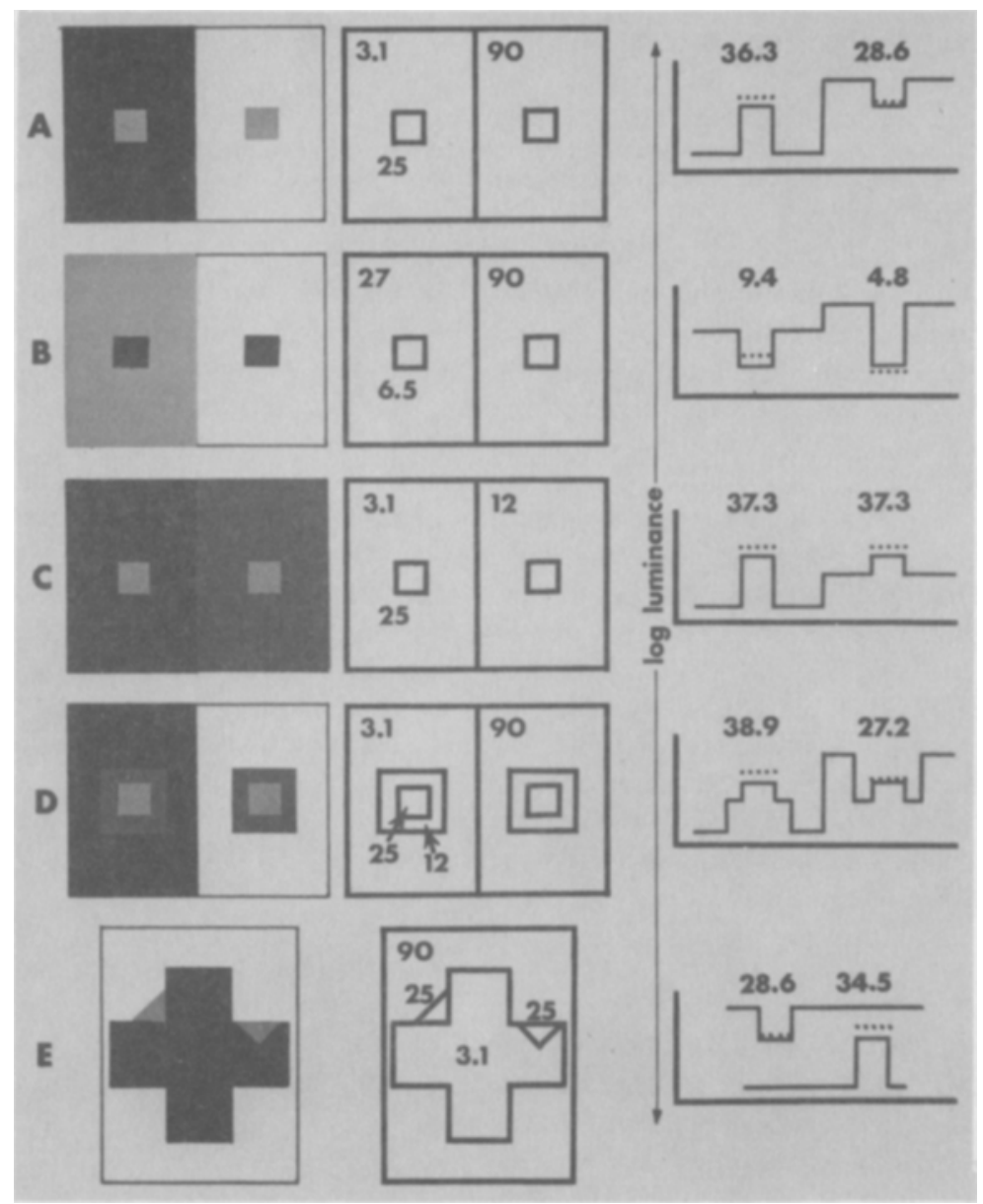

Figure 5. Appropriate edge-processing strategies for the constancy and contrast conditions of Experiment 1. (The term strategy is used here metaphorically. It is not intended as a mentalistic concept.) 
1 Munsell unit) is equal in the two cases. Inasmuch as both ratio matching and luminance matching are possible under the existing conditions, it may be that, even though only one operation is appropriate in a given situation, the possibility of each exerts a pull on the other, preventing its total success.

It is proposed that perceptual errors result from the presence of competing operations (one of which is inappropriate but both of which are possible) on edge relationships. The interested reader is urged to consider some closely related work reported in an insightful manner by Whittle and Challands (1969).

\section{Competing Operations in Earlier Studies}

A survey of some earlier studies in terms of the degree to which such competing operations were permitted provides additional, although indirect, support. The experiments of Hess and Pretori (1894/1970), Wallach (1948), Heinemann (1955), and Whittle and Challands (1969), for instance, can be ordered in this way. The display used by Hess and Pretori was very similar to that of the present Experiment 1, consisting of a side-by-side pair of target and surround fields. However, given the large range of luminances they used and the lack of any disambiguating context information, it would not have been clear whether the backgrounds differed in reflectance or in illumination. Evans (1948) reported that he replicated the Hess and Pretori display and found that it could be viewed in two quite different ways: either as two faces of a cube, with a smaller square in the center of each face, or as a display in the frontal plane, containing two small square apertures that opened onto a common uniform surface. Notice that these two ways are distinguished by whether the mediating edge appears as an illumination edge (at the corner of the cube) or a reflectance edge (in the coplanar view). One of these two attitudes had to be adopted by the observer. Evans (1948) stated:

The results obtained by asking the observer to take either one of these two attitudes were quite satisfying. The indeterminateness ceased at once. When the perception was that of a cube with gray squares the matches moved far in the direction of the ratios required by complete brightness constancy. When the perception was that of a uniformly illuminated rear surface seen through two apertures, the results moved equally far toward having identical intensities (luminances) on the two sides. (p. 166; italics added)

Here, as in the present Experiment 1, the observer was asked to equate the target squares. But two kinds of equality were possible: (1) the squares could be set equal in the sense of each relative to its own surround, or (2) they could be set equal in relation to a single common standard, say, the brighter of the two backgrounds. Depending on which attitude was appropriate, the mediating edge should have been either included in or excluded from the integration by which the target squares were perceptually compared.
Because Hess and Pretori (1894/1970) failed to distinguish between these two perceptual attitudes, with their attendant logical operations, it is not surprising that their data represent a compromise between ratio matching and luminance matching.

In the classic Wallach (1948) experiment, the two target-and-surround fields were spatially separated in a dark room. These conditions greatly reduce the observer's ability to make a luminance match, whereas the ability to make ratio matches is unaffected. Consequently Wallach obtained the first strong ratio results. But despite the remarkable cleanness of Wallach's data, it must be kept in mind that each of his studies produced small deviations from pure ratios and the deviations were almost always in the direction of luminance matches, which, although difficult to make under his conditions, nevertheless would have been just barely possible. Heinemann's (1955) results show the same pattern.

Whittle and Challands (1969) conducted an experiment that could be viewed as a replication of Wallach's (1948) experiment under conditions that completely prevented one of the competing operations. Their targets were square increments of light superimposed on larger background disks of light. They used a haploscopic method in which one target and its surrounding field was presented to one eye while the other was presented to the other eye, such that the outer boundaries of the background disks were in binocular registry. Since the target increments were shifted to opposite sides of the center of the background disk, the resulting binocular image consisted of the two target squares standing side by side (although not touching) on a single large background disk. The fact that the target squares appeared to stand on a common background (although they did not, physically) made it easy to make a precise match, and since the backgrounds were binocularly superimposed, they could not be compared. This prevented a luminance match based on the integration of all the edges, allowing only a ratio match. Whittle and Challands thus obtained virtually perfect ratio results with no systematic deviations in favor of a luminance match.

One possible test of the present hypothesis would be to present a side-by-side display, such as the traditional contrast display, under conditions that caused the mediating edge to disappear through retinal stabilization. The resulting appearance should produce a match completely defined by the edge ratio of each target. The targets, which would now appear on a homogeneous background, should appear black and white (respectively), although equal in luminance. Qualitative results by Yarbus $(1967$, p. 97) suggest that this does in fact occur.

\section{Two Types of Constancy}

There is another way to describe the relationship between contrast and constancy that could be very helpful. SLC also represents a failure of constancy in its own right, but a different kind of constancy. Surfaces not only appear constant under changes of illumination, they also ap- 
pear constant when viewed against different backgrounds (as pointed out by J. Walraven, personal communication, 1976, and Whittle \& Challands, 1969). Failures of this type of constancy are known as contrast effects, SLC being a particular instance. SLC has long received a great deal of attention, perhaps because it is both counterintuitive and easy to demonstrate. But the fact that a change in background produces a small change in the lightness of a surface has been allowed to eclipse the more important story, namely, the remarkable degree to which surfaces retain their lightness as the background changes.

Ironically, the same edge-ratio approach that so successfully solves the illumination-type constancy problem also reveals the background-type constancy problem, because edge ratios change so dramatically with background changes. The solution to the background-type constancy problem essentially lies in edge integration, but there seems to be an irreducible error in the integration process, as illustrated by SLC.

Recognizing that the visual system contends with two kinds of constancy, not just one, further highlights the symmetry between contrast and failures of constancy. It appears that the tendency toward each of these types of constancy weakens, or causes an error in, the other type.

\section{REFERENCES}

AREND, L. E. (1973). Spatial differential and integral operations in human vision: Implications of stabilized retinal image fading. Psychological Review, 8, 374-395.

Arend, L. E., Buehler, J. N., \& Lockhead, G. R. (1971). Difference information in brightness perception. Perception \& Psychophysics, 9, 367-370.

BARLOW, H. B. (1963). Slippage of contact lenses and other artefacts in relation to fading and regeneration of supposedly stable retinal images. Quarterly Journal of Experimental Psychology, 15, 36-51.

BENARY, W. (1924). Beobachtungen zu einem Experiment über Helligkeitskontrast. Psychologische Forschung, 5, 131-142.

Berman, P., \& Leibowitz, H. W. (1965). Some effects of contour on simultaneous brightness contrast. Journal of Experimental Psychology, 69, 251-256.

BURGH, P., \& GrINDLEY, G. C. (1962). Size of test patch and simultaneous contrast. Quarterly Journal of Experimental Psychology, 14, 89-93.

Cornsweet, T. N. (1970). Visual perception. New York: Academic Press.

Evans, R. M. (1948). An introduction to color. New York: Wiley.

FrEEMAN, R. B. (1967). Contrast interpretations of brightness constancy. Psychological Bulletin, 67, 165-187.

FRY, G. A., \& ALPERN, M. (1953). The effect of a peripheral glare source upon the apparent brightness of an object. Journal of the $O p$ tical Society of America, 43, 189-195.

GILCHRIST, A. (1979). The perception of surface whites and blacks. Scientific American, 24, 88-97.

Gilchrist, A. L., Delman, S., \& JACobsen, A. (1983). The classification and integration of edges as critical to the perception of reflectance and illumination. Perception \& Psychophysics, 33, 425-436.
Gilchrist, A. L., \& JACOBSEN, A. (1984). Perception of lightness and illumination in a world of one reflectance. Perception, 9, 936-944. HeinemanN, E. G. (1955). Simultaneous brightness induction as a function of inducing and test-field luminances. Journal of Experimental Psychology, 50, 89-96.

Helmholtz, H. voN. (1962). The recent progress in the theory of vision. In M. Kline (Ed.), Popular scientific lectures (pp. 93-185). New York: Dover. (Original work published 1868)

Helson, H. (1943). Some factors and implications of color constancy. Journal of the Optical Society of America, 33, 555-567.

Helson, H. (1964). Adaptation-level theory. New York: Harper \& Row.

Henneman, R. H. (1935). A photometric study of the perception of object color. Archiv für Psychologie, No. 179.

Hess, C., \& PRETORI, H. (1970). Quantitative investigation of the lawfulness of simultaneous brightness contrast (H. R. Flock \& J. H. Tenney, Trans.). Perceptual \& Motor Skills, 31, 947-969. (Original work published 1894)

JACOBSEN, A., \& Gilchrist, A. L. (1988a). Hess and Pretori revisited: Resolution of some old contradictions. Perception \& Psychophysics, 43, 7-14.

JACOBSEN, A., \& GiLChrist, A. L. (1988b). The ratio principle holds over a million-to-one range of illumination. Perception \& Psychophysics, 43, 1-6.

JAMESON, D., \& HURVICH, L. M. (1964). Theory of brightness and color contrast in human vision. Vision Research, 4, 135-154.

Johansson, G. (1964). Perception of motion and changing form. Scandanavian Journal of Psychology, 5, 181-208.

KATZ, D. (1935). The world of color (Trans. from 2nd German ed by R. B. MacLeod \& C. W. Fox). London: Kegan Paul, Trench, \& Trubner. (Original work published 1911)

KofFKA, K. (1935). Principles of Gestalt psychology. New York: Harcourt, Brace.

KRAUSKOPF, J. (1963). Effect of retinal image stabilization on the appearance of heterochromatic targets. Journal of the Optical Society of America, 53, 741-744.

LAND, E. H., \& MCCANN, J. J. (1971). Lightness and retinex theory. Journal of the Optical Society of America, 61, 1-11.

Leibowitz, H., Mote, F. A., \& Thurlow, W. R. (1953). Simultaneous contrast as a function of separation between test and inducing fields. Journal of Experimental Psychology, 46, 453-456.

LockE, N. M. (1935). Color constancy in the rhesus monkey and in man. Archiv für Psychologie, No. 193.

Mixesell, W. H., Bentley, M. (1930). Configuration and contrast Journal of Experimental Psychology, 13, 1-23.

RocK, I. (1983). The logic of perception. Cambridge, MA: MIT Press.

WALLACH, H. (1948). Brightness constancy and the nature of achromatic colors. Journal of Experimental Psychology, 38, 310-324.

WALLACH, H. (1976). Hans Wallach on perception. New York: Times Books.

Whittle, P., \& Challands, P. D. C. (1969). The effect of background luminance on the brightness of flashes. Vision Research, 9, 1095-1110.

Yarbus, A. L. (1967). Eye movements and vision. New York: Plenum Press.

\section{NOTE}

1. Land and McCann (1971) proposed a "random walk" method of edge integration that would account for some contrast patterns.

(Manuscript received July 10, 1983 revision accepted for publication October $13,1987$. ) 